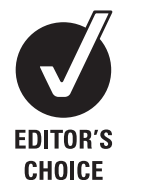

${ }^{1}$ Emergency Department, Royal Children's Hospital, Melbourne, Victoria, Australia

${ }^{2}$ Paediatric Cardiology Department, Oxford Radcliffe NHS Trust, Oxford, UK ${ }^{3}$ Murdoch Children's Research Institute, Melbourne, Victoria, Australia

${ }^{4}$ University of Melbourne, Melbourne, Victoria, Australia

\section{Correspondence to} Assoc Prof Franz E Babl, Paediatric Emergency Physician, Royal Children's Hospital, Melbourne, Victoria 3052, Australia;

franz.babl@rch.org.au

Accepted 19 August 2011 Published Online First 21 September 2011

\title{
Paediatric arrhythmias in the emergency department
}

\author{
Henning Clausen, ${ }^{1,2}$ Theane Theophilos, ${ }^{1,3}$ Kim Jackno, ${ }^{3}$ Franz E Babl ${ }^{1,3,4}$
}

\section{ABSTRACT}

Objective Emergency department (ED) staff need to rapidly establish accurate diagnosis and management for children with arrhythmias. Limited data are available on the presenting features, epidemiology and management of arrhythmias encountered in the ED. The aim of this study was to characterise the incidence, presenting features, management and outcomes of arrhythmias at a large tertiary children's hospital ED.

Patients and methods Retrospective review of medical records identified via the ED electronic database using ICD-10 codes for arrhythmias including cardiac arrests over a 6-year period (2002-2008). Patients $<18$ years were analysed using predefined criteria.

Results There were a total of 444 non-arrest arrhythmias with an incidence of 11.5:10000 presentations. Median age of patients at presentation was 10.4 years; $45 \%$ were male. Supraventricular arrhythmias (SVTs) represented the largest subgroup ( $n=250,56 \%)$. Conduction disorders $(n=18)$, ventricular tachycardia $(n=17)$ and atrial flutter/fibrillation $(n=7)$ were rare. There were 19 cardiac arrests. Fifty-seven (13\%) patients had underlying congenital heart disease. For ongoing SVT $(n=135)$, vagal manoeuvres were used in $74 \%$, and antiarrhythmic drugs in 64\%. In five patients with SVT, drugs other than adenosine were used. Defibrillators were used only on 2 occasions for arrthymias and 6 times for cardiac arrests. 18 of 19 children in cardiac arrest died. Conclusion In this largest paediatric series outside the intensive care and postoperative setting, arrhythmias were uncommon, defibrillator use was very rare, and observed mortality was low.

\section{INTRODUCTION}

The emergency department (ED) will often be the first port of call for children with palpitations or chest pain caused by an irregular heartbeat. The background of the concerns with these children is the possibility of fatal arrhythmias which have been recognised in the aetiology of out-of-hospital cardiac arrests. ${ }^{12}$ Children with congenital heart disease in particular are at increased risk of significant arrhythmias and sudden cardiac death. ${ }^{3}$ Nonsustained ventricular and supraventricular tachycardias (VTs and SVTs) are commonly encountered during the acute postoperative phase in the paediatric intensive care unit (PICU). ${ }^{4}$ However, even otherwise healthy children may experience arrhythmia-related syncopes or sudden death. ${ }^{5}$ Sudden cardiac death is encountered relatively often in adult patients with increased risk due to underlying structural heart disease or myocardial ischaemia. ${ }^{6-10}$

Comparatively few data have been published on the epidemiology, initial emergency management and outcome of paediatric arrhythmias in the ED. ${ }^{11-13}$ Management of arrhythmias and cardiac arrests in children has been addressed by the International Liaison Committee on Resuscitation (ILCOR). ${ }^{14}$ Training courses, such as Advanced Paediatric Life Support (APLS), ${ }^{15}$ include sections on the drug and electrical management of arrhythmias. Whether this leads to better outcomes remains controversial. ${ }^{16}$

We set out to characterise the epidemiology of arrhythmias in children in a large tertiary paediatric ED and aimed to identify the pattern of use of antiarrhythmic agents and defibrillators. Ultimately, these data should assist staff to optimally prepare for cardiac arrhythmias in children.

\section{PATIENTS AND METHODS \\ Design and setting}

A retrospective observational study was conducted in the ED of a large, urban children's hospital with an annual ED census of 69000 patients. All children $<18$ years of age presenting with cardiac arrhythmia or cardiac arrest between 1 January 2002 and 30 August 2008 were included. This study was approved as an audit by the hospital institutional review board.

\section{Inclusion and exclusion criteria}

Arrhythmia patients were identified using ICD-10 codes (I45.9, I46.9, I47.1, I47.2, I48, I49.9 or Z95) via the ED Hospital Administration System where a cardiac arrhythmia comprised the primary diagnosis. Sinus tachycardia due to fever, dehydration or respiratory complaints, as derived from the individual case notes, were excluded. Patients coded with cardiac arrest were analysed separately. We excluded trauma-related arrests and those triaged as dead on arrival.

\section{Procedure}

For identified cases, patient's written case notes and electronic records were extracted for demographics, arrhythmia information, medical history, signs and symptoms, ED and inpatient or outpatient management, and patient outcomes. We recoded arrhythmias based on (in descending order) documented electrophysiological testing, cardiology consultation, $24 \mathrm{~h}$ ECG recordings, ECG analysis in the $\mathrm{ED}$, and diagnoses documented by the treating physician. When more than one type of arrhythmia was present or when there was a divergent interpretation of the data, we reanalysed the primary sources of arrhythmia assessment to establish the most serious and accurate type of arrhythmia for our analysis. Cardiology or ED physician assessment was otherwise accepted as stated.

\section{Definitions}

ED triage urgency was assessed using the national Australasian triage scale (ATS). ${ }^{17}$ Patients with ATS 
category 1, 2, 3, 4 and 5 are to be seen immediately or within 10, 30, 60 and $120 \mathrm{~min}$, respectively. Sinus bradycardia was defined as a heart rate of $<60$ per $\min$.

\section{Analysis}

Values are reported as mean with SD or median with IOR. Relevant percentages are reported with 95\% CIs.

\section{RESULTS}

Search by ICD-10 codes identified 523 ED visits with a discharge diagnosis of arrhythmias during the 6.5-year study period. We excluded 79 encounters with duplicative documentation and those coded as paediatric arrhythmias but which on review of the case notes did not meet our inclusion criteria. There were therefore a total of 444 patient encounters with arrhythmias in 288 individual patients, which constituted the study group for further analysis. We identified 19 cases of cardiac arrest during the study period.

Paediatric arrhythmias presented with an incidence of 11.5/ 10000 ED presentations. SVT occurred in 6.5/10000 presentations. Other specific arrhythmias and cardiac arrest were very uncommon (tables 1 and 2). Among the presenting complaints, palpitations were named most often (61\%). Congenital heart disease was noted in $13 \%$ of patients. Thirty-one per cent of patients required admission, and only 16 patients (3.5\%) were admitted to the PICU.

\section{Supraventricular tachycardias}

There were 250 ED presentations with SVT in 135 patients (table 3). Median age was 8.8 years. The majority (63\%) presented only once (figure 1). One-third of SVT patients were admitted to hospital, five to the PICU. One-third of SVT patients had ventricular pre-excitation as documented in their records. Two-thirds of patients presented with palpitations. Half the patients $(135 ; 54 \%)$ were in active SVT on arrival. Attempted vagal manoeuvres were documented in 100 of 135 presentations (74.1\%) with persistent SVT. In 86 of 135 (64\%) with active SVT antiarrhythmic agents were administered. Adenosine was the agent most often used in 82 of 86 cases $(95 \%)$ with a median number of two adenosine doses (IOR $1-2$, range $1-7$ ). Thirtynine of 82 patients (48\%) reverted to sinus rhythm after a single dose of adenosine, 25 (30\%) after two doses.

Only six patients with SVT received other treatments including amiodarone, sotalol, propranolol and cardioversion. Three patients received amiodarone infusions. Two patients failed multiple doses of adenosine and reverted with amiodarone. One patient with known congenital heart disease and atrial tachycardia received amiodarone in the $\mathrm{ED}$, who was subsequently monitored in the PICU. A 15-year-old with repaired atrioventricular septal defect presented with SVT and received metaraminol for significant hypotension before hospital admission. On arrival in the ED, he was started on sotalol as per

Table 1 Incidence of arrhythmias in the emergency department

\begin{tabular}{lrl}
\hline & N & Per 10000 visits \\
\hline Arrhythmia (any) & 444 & 11.5 \\
SVT & 250 & 6.5 \\
Conduction disorder & 18 & 0.5 \\
VT & 17 & 0.5 \\
AF & 7 & 0.2 \\
Cardiac arrest & 19 & 0.5 \\
\hline
\end{tabular}

$\mathrm{AF}$, atrial fibrillation/flutter; SVT, supraventricular tachycardia; VT, ventricular tachycardia. cardiology advice. A 1-day-old term baby was transferred from another hospital by the neonatal retrieval service with an antenatal diagnosis of SVT. After unsuccessful adenosine and propranolol treatment before arrival in the $\mathrm{ED}$, the heart rate was recorded as 250 beats/min in the ED. Propranolol was continued, and the patient was admitted with persistent reciprocating junctional tachycardia for further cardiac management. Only one SVT patient with known pre-excitation was successfully cardioverted in the ED after unsuccessful vagal manoeuvres and adenosine.

\section{Conduction disorders}

Conduction disorders were seen in 18 children aged 9 months to 17 years. Atrioventricular (AV) conduction disorders were found in four children with a first degree AV block, four with a second degree AV block, and four with complete AV block. Four of these 12 children were known to have a degree of AV conduction delay before attending the ED. An additional six patients with long OT syndrome were seen. Half of these children had a family history of channelopathies. Two of these were asymptomatic at presentation and specifically referred to the ED by their family physician for evaluation. Other ED presentations of patients with conduction disorders were related to chest pain (six), lethargy (four), syncope (three), respiratory distress (two) and palpitations (one). One syncopal patient with known complete AV block received isoprenaline for a failing pacemaker that was replaced during hospital admission. Another patient with a pacemaker presenting with complete heart block received a new device during admission. One patient with second degree AV block was diagnosed with rheumatic fever and started on penicillin and aspirin. All children presented only once during our observation period, and no other antiarrhythmic agents or electrical interventions were administered in ED.

\section{Ventricular tachycardias}

There were 17 episodes of VT in only five patients, aged 10-16 years. One patient with known torsade des pointes tachycardia and implantable cardioverter defibrillator (ICD) in situ presented 10 times during the observation period following shocks delivered by the device. Another patient with Brugada syndrome who had an ICD presented on four occasions with VT, as documented by the device. Recurrent idiopathic VTwas seen in one patient, and cardiomyopathy caused VT in another case. One child presented with VT and a positive family history of ventricular arrhythmias. Syncope was the leading cause of presentation (10), followed by respiratory distress (three), palpitations (two), chest pain (one) and lethargy (one). Only one patient had an active ED intervention: a 16-year-old with known cardiomyopathy presented with palpitations. $\mathrm{He}$ was treated for VT with lignocaine before hospital admission. On arrival, his VT persisted with initially stable haemodynamics. He was given amiodarone with no success and was subsequently intubated and cardioverted to sinus rhythm for increasing cardiovascular instability. He received an ICD during his hospital stay.

\section{Atrial fibrillation/flutter}

AF was coded in seven children who each presented once to ED. These patients were aged 12-17 years, and six of them had known AF either associated with dilated cardiomyopathy (CMP) (one) or operated complex congenital heart disease (five). Only one patient had de novo AF and was diagnosed as having Brugada syndrome during admission. Patients presented with palpitations (four), chest pain (two) or lethargy (one). None of 
Table 2 Patient demographics of all episodes of arrhythmia $(n=444)$

\begin{tabular}{lcc}
\hline Arrhythmia diagnosis & Presentations, $\mathbf{n ~ ( \% ) ~}$ & Individuals, n (\%) \\
\hline SVT & $250(56.3)$ & $135(46.9)$ \\
Conduction disorder & $18(4.1)$ & $18(6.3)$ \\
VT & $17(3.8)$ & $5(1.7)$ \\
AF & $7(1.6)$ & $6(2.1)$ \\
Other arrhythmias & $151(34.0)$ & $124(43.1)$ \\
$\quad$ Sinus arrhythmia & $134(30.2)$ & $111(38.5)$ \\
Sinus bradycardia & $10(2.3)$ & $7(2.4)$ \\
$\quad$ Premature atrial contractions & $3(0.7)$ & $3(1.0)$ \\
$\quad$ Premature ventricular contractions & $1(0.2)$ & $1(0.4)$ \\
Total & $444(100)$ & $288(100)$ \\
\hline
\end{tabular}

\begin{tabular}{ll}
\hline Demographic/symptoms/outcome & Value \\
\hline Age (years) & \\
Median & 10.4 \\
IQR & $6.4-14.1$ \\
$\quad$ Range & $0-17.9$ \\
Age categories (n (\%)) & \\
$\quad<1$ & $47(10.6)$ \\
$1-5$ & $62(14.0)$ \\
$6-12$ & $193(43.5)$ \\
$13-17$ & $142(32.0)$ \\
Gender (n (\%)) & \\
Male & $199(44.8)$ \\
Female & $245(55.2)$ \\
Triage categories (n (\%)) & \\
1 & $16(3.6)$ \\
2 & $150(33.8)$ \\
3 & $191(43.0)$ \\
4 & $81(18.2)$ \\
5 & $6(1.4)$ \\
Arrival mode (n (\%)
\end{tabular}

Arrival mode $(\mathrm{n}(\%))^{*}$

Car/walk

Ambulance

Air ambulance

Helicopter

Primary presenting complaint $(\mathrm{n}(\%)) \dagger$

Palpitations

Chest pain

Lethargy

Syncope

Respiratory distress

Intoxication

Presenting complaints (multiple possible) (n (\%))

Palpitations

Chest pain

Lethargy

Respiratory distress

Syncope

Dizziness

Chest tightness

Seizure

Unresponsive

Intoxication

Other

Cardiac history (n (\%))

Congenital heart disease $\neq$

Known arrhythmia or other

cardiovascular disease§

Discharge destination from ED (n (\%))

Discharge home

Discharge to ward

Discharge to PICU
Table 2 Continued

\begin{tabular}{ll}
\hline Demographic/symptoms/outcome & Value \\
\hline Length of admission (days) & \\
Median & 2 \\
IOR & $1-4$ \\
Range & $0-34$ \\
\hline
\end{tabular}

*Two missing values.

†Eight missing values.

$\ddagger$ Two missing values.

$\S$ Two missing values.

SVT, supraventricular tachycardia; VT, ventricular tachycardia; AF, atrial fibrillation/flutter.

the children received antiarrhythmic drugs or electrical intervention in the ED. Six patients were admitted; four were cardioverted during hospitalisation.

\section{Other arrhythmias}

There were a total of 151 presentations classified as 'other arrhythmias' which were sinus arrhythmia in 134 cases, sinus bradycardia with a heart rate of $<60$ beats/min in 10 cases, sinus tachycardia without fever, dehydration or respiratory compromise and premature atrial contractions in three cases, and one case of frequent ventricular ectopics. The median age was 10.9 years (IOR 7.9-14.8) in this subgroup with the majority of children 6 years or older $(87 \%)$. Palpitations were the most common complaint. None received antiarrhythmic drugs in the ED. The admission rate was $20 \%$, with one admission to the PICU following a caffeine overdose with numerous 'Red Bull' drinks and caffeine tablets in a 14-year-old.

\section{Cardiac arrests}

During the study period, we encountered 19 cardiac arrests presenting to the ED. According to the first ECG tracing in the $\mathrm{ED}$, pulseless electrical activity was recorded in 11, ventricular fibrillation (VF) in five, sinus bradycardia in two, and AF leading to fast ventricular conduction in an 11-year-old boy with Wolff-Parkinson-White syndrome. He was defibrillated to a stable sinus rhythm and underwent electrophysiological testing with ablation of his accessory pathway. He was the only survivor to hospital discharge in this cohort. Among four affected children with known operated congenital heart disease, two had shunt-dependent cyanotic lesions, one had recent surgery for aortic regurgitation, and one had undergone corrective surgery for pulmonary atresia with ventricular septal defect and major aortopulmonary collaterals. One patient had known coronary aneurysms following Kawasaki's disease. Another one was found to have cardiomyopathy on post-mortem examination. Three children with overwhelming sepsis and two with end-stage renal failure were seen. One patient was diagnosed as having glutaric acidaemia type 1 on post-mortem examination, and another one was diagnosed as having a previously unrecognised congenital diaphragmatic hernia. One patient suffered respiratory failure with subsequent cardiac arrest in the out-ofhospital setting. One teenager with cocaine intoxication died in the ED. Two children were diagnosed as having sudden infant death syndrome (SIDS) on post-mortem examination, and two others had out-of-hospital arrests of unknown aetiology. Sixteen of these patients received epinephrine, 11 atropine, one metaraminol, one amiodarone, one esmolol and one magnesium. Four patients were defibrillated before hospital admission and six in the ED, which included the one survivor. Five patients had VF as the first documented rhythm in the $\mathrm{ED}$, and four were defibrillated to temporary sinus rhythm (one cocaine overdose, one end-stage renal failure, one SIDS, one out-of-hospital arrest of unknown aetiology) with subsequent degeneration of 
Table 3 Supraventricular tachycardia (SVT): patient demographics, interventions and outcomes $(n=250)$

\begin{tabular}{|c|c|}
\hline Demographic/symptoms/intervention/outcome & Value \\
\hline \multicolumn{2}{|l|}{ Age (years) } \\
\hline Median & 8.8 \\
\hline IQR & $3.6-12.5$ \\
\hline Range & $0-17.5$ \\
\hline \multicolumn{2}{|l|}{ Age categories (n (\%)) } \\
\hline$<1$ & $42(16.8)$ \\
\hline $1-5$ & $45(18.0)$ \\
\hline $6-12$ & $108(43.2)$ \\
\hline $13-17$ & $55(22.0)$ \\
\hline \multicolumn{2}{|l|}{ Gender (n (\%)) } \\
\hline Male & $101(40.4)$ \\
\hline Female & $149(59.6)$ \\
\hline \multicolumn{2}{|l|}{ Cardiac history (n (\%)) } \\
\hline Congenital heart disease & $28(11.2)$ \\
\hline Known arrhythmia or other cardiovascular disease & $197(78.8)$ \\
\hline Ventricular pre-excitation* & $72(32.7)$ \\
\hline \multicolumn{2}{|l|}{ Triage categories (n (\%)) } \\
\hline 1 & $16(6.4)$ \\
\hline 2 & $122(48.8)$ \\
\hline 3 & $88(35.2)$ \\
\hline 4 & $22(8.8)$ \\
\hline 5 & $2(0.8)$ \\
\hline \multicolumn{2}{|l|}{ Arrival mode (n (\%)) } \\
\hline Car/walk & $156(62.9)$ \\
\hline Ambulance & $91(36.7)$ \\
\hline Helicopter & $1(0.4)$ \\
\hline \multicolumn{2}{|l|}{ Primary presenting complaint $(\mathrm{n}(\%)) \dagger$} \\
\hline Palpitations & $146(58.4)$ \\
\hline Chest pain & $42(16.8)$ \\
\hline Lethargy & $36(14.4)$ \\
\hline Respiratory distress & $18(7.2)$ \\
\hline Syncope & $6(2.4)$ \\
\hline \multicolumn{2}{|l|}{ Presenting complaints (multiple possible) (n (\%)) } \\
\hline Palpitations & $157(65.2)$ \\
\hline Chest pain & $48(20.0)$ \\
\hline Lethargy & $45(18.7)$ \\
\hline Respiratory distress & $34(14.1)$ \\
\hline Dizziness & $14(5.8)$ \\
\hline Syncope & $6(2.5)$ \\
\hline Chest tightness & $6(2.5)$ \\
\hline Seizure & $1(0.4)$ \\
\hline Other & $55(22.8)$ \\
\hline \multicolumn{2}{|l|}{ SVT status on arrival $(\mathrm{n}(\%))$} \\
\hline Resolved before ED arrival & $115(46.0)$ \\
\hline Active SVT on arrival & $135(54)$ \\
\hline \multicolumn{2}{|l|}{ Interventions in ED ( $\mathrm{n}(\%)$ ) } \\
\hline Vagal manoeuvres attempted $\neq$ & $100(46.9)$ \\
\hline Antiarrhythmic medication administered & $85(34.0)$ \\
\hline Adenosine & $82(32.8)$ \\
\hline Amiodarone & $3(1.2)$ \\
\hline Sotalol & $1(0.4)$ \\
\hline Propranolol & $1(0.4)$ \\
\hline Cardioversion & $1(0.4)$ \\
\hline \multicolumn{2}{|l|}{ Discharge destination from ED (n (\%)) } \\
\hline Discharge home & $171(68.4)$ \\
\hline Discharge to ward & $74(29.6)$ \\
\hline Discharge to PICU & $5(2.0)$ \\
\hline \multicolumn{2}{|l|}{ Length of admission (days) } \\
\hline Median & 3 \\
\hline IOR & $1-5$ \\
\hline Range & $0-34$ \\
\hline
\end{tabular}

*30 missing values.

†Two Missing values.

$\neq 37$ missing values.

$E D$, emergency department; ICU, intensive care unit.

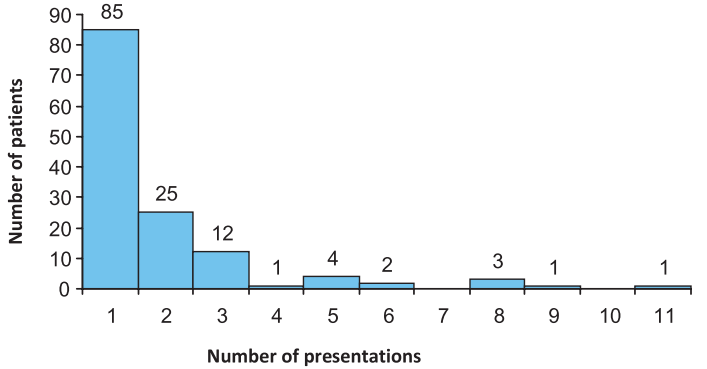

Figure 1 Frequency of presentations $(n=250)$ in patients with supraventricular tachycardia $(n=135)$.

cardiac rhythm or irreversible severe end organ damage leading to death. One SIDS patient with VF did not respond to defibrillation.

\section{DISCUSSION}

In this largest ED series to date, paediatric arrhythmias were dominated by SVTs. Few patients had other significant arrhythmias beyond sinus arrhythmias. Very few patients were cardiovascularly unstable or died from arrhythmias. While adenosine was commonly used in SVT, other antiarrhythmics were only used in seven of 444 patients (2\%) with any arrhythmias outside cardiac arrest. Potentially shockable rhythms such as unstable SVT, VT and VF were only encountered very rarely. Over 6.5 years at this large Australian paediatric $\mathrm{ED}$, a defibrillator was only used in one patient with unstable SVT and in one patient with VT-that is, $0.5 \%$ of patients outside cardiac arrest, and six of 19 patients with cardiac arrests in the ED.

The only comparable dataset on the epidemiology of paediatric arrhythmias was a review of data from 26 regional EDs in the USA by Sacchetti et al. ${ }^{11}$ As none of the EDs in that study had a designated paediatric ED and our study ED was located at the only tertiary regional children's hospital for a population base of five million, we expected much higher numbers of arrhythmias and patients receiving complex antiarrhythmic or electrical therapy. However, overall, we found the distribution of the types of arrhythmias only slightly different from the Sacchetti et al data. ${ }^{11}$ Whereas sinus arrhythmia was most common in the analysis by Sacchetti et al, we saw SVT as the predominant arrhythmia. Even though this study included more patients with congenital heart disease $(13 \%)$ than that of Sacchetti et al $(2 \%)$, the incidence of ventricular, cardiovascularly unstable and fatal arrhythmias was low. These data are in contrast with the postcardiac surgery and PICU setting, where high rates of ventricular arrhythmias and higher mortality have been reported, ${ }^{418}$ and in contrast with adult data. ${ }^{19}$

A number of children had been coded as SVT, but did not present with ongoing tachycardia in the ED. Some of these children, or their parents, had been previously instructed to attempt vagal manoeuvres and managed to terminate the arrhythmia before the ED visit while others stopped spontaneously. Many children had a history of SVT, which helped in establishing a rapid treatment plan. Vagal manoeuvres are to some extent beneficial when the patient is haemodynamically stable. $^{1420}$ We note that, of 135 with active SVT, 100 had documented vagal manoeuvres, of which 82 went on to receive adenosine, which would indicate a low rate of success of vagal manoeuvres of any kind. We are unable to address the optimal 
mode of vagal stimulation because of a lack of detailed ED documentation. Adenosine was the most effective treatment of choice for SVT as described in other studies ${ }^{21} 22$ and as suggested by ILCOR. ${ }^{14}$ Only a minority required other drug treatments, such as amiodarone, sotalol or propranolol. ${ }^{23} 24$

The majority of children who could talk described 'palpitations' as the leading symptom for their SVT. 'Chest pain' was ranked second, followed by syncopal events in the ED records. Coexisting congenital heart disease was seen in more than $10 \%$ of SVT cases, and ventricular pre-excitation was diagnosed in one-third of presentations. Haemodynamic instability was rare in the SVT group, and only one patient with known Wolff-Parkinson-White syndrome experienced a cardiac arrest secondary to atrial fibrillation with subsequent fast ventricular conduction and loss of cardiac output. Electric shocks were successful in establishing spontaneous circulation in this case, and the patient made a full recovery. There was a distinct age peak for SVT presentations in children 6-12 years of age, which stands in contrast with previous reports. ${ }^{11}$ Although we did not encounter any in-hospital deaths in infants presenting with SVT during the 6.5-year study period, unrecognised SVT may lead to acute cardiac decompensation in this age group. ${ }^{25} 26$

Arrhythmias beyond SVT, including conduction disorders, AF and VTs were very rare and mostly presented with prior and known structural or non-structural cardiac history. Even this more complex subset rarely required antiarrhythmic or electrical therapy in the ED. This again stands in contrast with postcardiac surgery, PICU and the adult setting. ${ }^{4} 1819$ The number of cardiac arrests seen in the ED was also low, and the aetiologies were diverse with a poor overall survival rate as described in previous reports. ${ }^{27-29}$ Only one out of 19 children survived to discharge. Non-shockable rhythms were seen in the majority of children, and defibrillators were used for only six patients. Epinephrine was the drug most commonly used. Resuscitation management was overall consistent with published paediatric consensus guidelines. ${ }^{14} 23$

ED staff education and clinical practice guidelines will need to consider these findings. The low frequency of unstable arrhythmias and urgent antiarrhythmic and electrical interventions in the ED, with the exception of adenosine for SVT, will likely pose challenges for staff training and skills retention. Antiarrhythmic management including defibrillator use may require the use of simulation-based training to maintain rarely used skills, ${ }^{30} 31$ and complex or unresponsive arrhythmias should lead to the early involvement of, and consultation with, cardiology and intensive care services.

\section{Limitations}

This study has a number of limitations. The case identification was based on ICD-10 codes of arrhythmia as set out in the patients and methods section. Patients would have been missed if their presentations were coded with a non-arrhythmia ED diagnosis. Management represents local practice, and the availability or absence of intensive care and cardiology services will influence whether certain interventions are performed in the ED or elsewhere. Local paramedic services at the study hospital can terminate resuscitation efforts before hospital admission, which may have influenced the distribution of presenting rhythms in patients with cardiac arrest. Patients triaged dead on arrival and trauma-related cardiac arrests were excluded. As a retrospective study, data abstraction depended on the accuracy and completeness of the medical records. We attempted to optimise chart review as set out by Gilbert et al. ${ }^{32}$ However, the abstractors were not blinded to the study hypothesis.

\section{What is already known on this subject}

Arrhythmias may require rapid interventions by emergency physicians.

- Education and training programmes emphasise antiarrhythmic and defibrillator use.

- Yet, data on epidemiology and interventions in arrhythmias outside the intensive care and cardiology setting are scarce.

\section{What this study adds}

In a large emergency department dataset, unstable or fatal arrhythmias were very rare.

- Beyond adenosine, antiarrhythmics or a defibrillator were used very rarely for arrhythmia management.

\section{CONCLUSION}

In this large paediatric series, cardiovascularly unstable or fatal arrhythmias were very rare in the ED. Beyond adenosine for SVT, antiarrhythmic agents or a defibrillator were rarely used. These findings have implications for staff education and skill retention.

Acknowledgements We thank Vanessa Gorman who assisted with data extraction. We acknowledge grant support from the Murdoch Children's Research Institute, Melbourne, Australia.

Funding Murdoch Children's Research Institute.

Competing interests None.

Ethics approval Royal Children's Hospital, Melbourne.

Contributors HC: design, methods, ethics, data acquisition, analysis, data interpretation, drafting. TT: methods, analysis. KJ: data acquisition, analysis. FEB: design, methods, ethics, data interpretation, drafting.

Provenance and peer review Not commissioned; externally peer reviewed.

\section{REFERENCES}

1. Topijan AA, Nadkarni VM, Berg RA. Cardiopulmonary resuscitation in children. Curr Opin Crit Care 2009;15:203-8.

2. Wren C. Sudden death in children and adolescents. Heart 2002:88:426-31.

3. Silka MJ, Hardy BG, Menashe VD, et al. A population-based prospective evaluation of risk of sudden cardiac death after operation for common congenital heart defects. J Am Coll Cardiol 1998;32:245-51.

4. Hoffman TM, Wernovsky G, Wieand TS, et al. The incidence of arrhythmias in a pediatric cardiac intensive care unit. Pediatr Cardiol 2002;23:598-604.

5. Liberthson RR. Sudden death from cardiac causes in children and young adults. N Engl J Med 1996;334:1039-44.

6. Zheng ZJ, Croft JB, Giles WH, et al. Sudden cardiac death in the United States, 1989 to 1998. Circulation 2001;104:2158.

7. Chugh SS, Jui J, Gunson K, et al. Current burden of sudden cardiac death: multiple source surveillance versus retrospective death certificate-based review in a large U.S. community. J Am Coll Cardiol 2004;44:1268.

8. Zipes DP, Camm AC, Borggrefe M, et al. ACC/AHA/ESC 2006 Guidelines for Management of Patients With Ventricular Arrhythmias and the Prevention of Sudden Cardiac Death: A Report of the American College of Cardiology/American Heart Association Task Force and the European Society of Cardiology Committee for Practice Guidelines (Writing Committee to Develop Guidelines for Management of Patients With Ventricular Arrhythmias and the Prevention of Sudden Cardiac Death) Developed in Collaboration With the European Heart Rhythm Association and the Heart Rhythm Society. Circulation 2006;114:e385-484.

9. Kuller LH. Sudden death-definition and epidemiologic considerations. Prog Cardiovasc Dis 1980;23:1.

10. Kannel WB, Doyle JT, McNamara PM, et al. Precursors of sudden coronary death Factors related to the incidence of sudden death. Circulation 1975;51:606.

11. Sacchetti A, Moyer V, Baricella R, et al. Primary cardiac arrhythmias in children. Pediatr Emerg Care 1999;15:95-8. 
12. Perry JC. The diagnosis and emergency management of tachyarrhythmias. Pediatr Emerg Med Rep 1996;1:65-73.

13. Cetta F, Bell TJ, Ros SP. Emergency department evaluation of an infant with chaotic atrial tachycardia. Pediatr Emerg Care 1997:13:120-2.

14. de Caen AR, Kleinman ME, Chameides L, et al. Part 10: Paediatric basic and advanced life support: 2010 International Consensus on Cardiopulmonary Resuscitation and Emergency Cardiovascular Care Science with Treatment Recommendations. Resuscitation 2010;81(Suppl 1):e213-59.

15. Advanced Life Support Group. Advanced pediatric life support: the practical approach. In: Martin S, Susan W, eds. 5th edn. Chichester, UK: Wiley Blackwell, 2011.

16. Arshid M, Lo TY, Reynolds F. Quality of cardio-pulmonary resuscitation (CPR) during paediatric resuscitation training: time to stop the blind leading the blind. Resuscitation 2009;80:558-60.

17. Australasian College of Emergency Medicine. Policy on the Australasian Triage Scale. http://www.acem.org.au/media/policies_and_guidelines/ P06 Aust Triage Scale - Nov 2000.pdf (accessed 13 Jul 2011).

18. Kamel $\mathbf{Y} \overline{\mathbf{H}}$, Sewielam $\overline{\mathbf{M}}$. Arrhythmias as early post-operative complications of cardiac surgery in children at Cairo University. J Med Sci 2009;9:126-32.

19. Goldberger JJ, Cain ME, Hohnloser SH, et al. American Heart Association, American College of Cardiology Foundation, Heart Rhythm Society. American Heart Association/American College of Cardiology Foundation/Heart Rhythm Society scientific statement on noninvasive risk stratification techniques for identifying patients at risk for sudden cardiac death: a scientific statement from the American Heart Association Council on Clinical Cardiology Committee on Electrocardiography and Arrhythmias and Council on Epidemiology and Prevention. Circulation 2008;118:1497-518.

20. Wen ZC, Chen SA, Tai CT, et al. Electrophysiological mechanisms and determinants of vagal manoeuvres for termination of paroxysmal supraventricular tachycardia. Circulation 1998;98:2716-23.

21. Overholt ED, Rheuban KS, Gutgesell HP, et al. Usefulness of adenosine for arrhythmias in infants and children. Am J Cardiol 1988;61:336.
22. Paul T, Bertram H, Bokenkamp R, et al. Supraventricular tachycardia in infants, children and adolescents: diagnosis, and pharmacological and interventional therapy. Paediatr Drugs 2000;2:171.

23. Australian Resuscitation Council. Paediatric advanced life support: Australian Resuscitation Council Guidelines 2006. Emerg Med Australas 2006;18:357-71.

24. Bianconi L, Castro A, Dinelli $\mathrm{M}$, et al. Comparison of intravenously administered dofetilide versus amiodarone in the acute termination of atrial fibrillation and flutter A multicentre, randomized, double-blind, placebo-controlled study. Eur Heart $J$ 2000;21:1265-73.

25. Doniger SJ, Sharieff GQ. Pediatric dysrhythmias. Pediatr Clin N Am 2006; 53:85-105.

26. Gilljam T, Jaeggi E, Gow RM. Neonatal supraventricular tachycardia: outcomes ove a 27-year period at a single institution. Acta Paediatrica 2008:97:1035-9.

27. Schindler MB, Bohn D, Cox PN, et al. Outcome of out-of-hospital cardiac or respiratory arrest in children. N Engl J Med 1996;335:1473-9.

28. López-Herce J, García C, Domínguez P, et al. Spanish Study Group of Cardiopulmonary Arrest in Children. Characteristics and outcome of cardiorespiratory arrest in children. Resuscitation 2004;63:311-20.

29. Atkins DL, Everson-Stewart S, Sears GK et al. Resuscitation Outcomes Consortium Investigators. Epidemiology and outcomes from out-of-hospital cardiac arrest in children: the Resuscitation Outcomes Consortium Epistry-Cardiac Arrest. Circulation 2009:119:1484-91.

30. Donoghue A, Nishisaki A, Sutton R, et al. Reliability and validity of a scoring instrument for clinical performance during Pediatric Advanced Life Support simulation scenarios. Resuscitation 2010;81:331-6.

31. Hunt EA, Walker AR, Shaffner DH, et al. Simulation of in-hospital pediatric medical emergencies and cardiopulmonary arrests: highlighting the importance of the first 5 minutes. Pediatrics 2008;121:e34-43.

32. Gilbert EH, Lowenstein SR, Koziol-McLain J, et al. Chart reviews in emergency medicine research: Where are the methods? Ann Emerg Med 1996:27:305-8. 